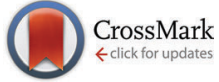

Cite this: J. Mater. Chem. C, 2016, 4, 3765

\title{
Benzobisoxazole cruciforms: a tunable, cross- conjugated platform for the generation of deep blue OLED materials $\uparrow$
}

\author{
Ramiro Chavez III, ${ }^{a}$ Min Cai, ${ }^{b}$ Brian Tlach, ${ }^{a}$ David L. Wheeler, ${ }^{c}$ Rajiv Kaudal, ${ }^{b}$ \\ Ayuna Tsyrenova, ${ }^{\text {bd }}$ Aimée L. Tomlinson, ${ }^{c}$ Ruth Shinar, ${ }^{e}$ Joseph Shinar ${ }^{\mathrm{b}}$ and \\ Malika Jeffries-EL*a
}

\begin{abstract}
Four new cross-conjugated small molecules based on a central benzo[1,2-d:4,5- $\left.d^{\prime}\right]$ bisoxazole moiety possessing semi-independently tunable HOMO and LUMO levels were synthesized and the properties of these materials were evaluated experimentally and theoretically. The molecules were thermally stable with $5 \%$ weight loss occurring well above $350{ }^{\circ} \mathrm{C}$. The cruciforms all exhibited blue emission in solution ranging from 433-450 $\mathrm{nm}$. Host-guest OLEDs fabricated from various concentrations of these materials using the small molecule host 4,4'-bis(9-carbazolyl)-biphenyl (CBP) exhibited deep blue-emission with Commission Internationale de L'Eclairage (CIE) coordinates of $(0.15 \leq x \leq 0.17,0.05 \leq y \leq 0.11)$, and maximum luminance efficiencies as high as $\sim 2 \mathrm{~cd} \mathrm{~A}^{-1}$. These results demonstrate the potential of benzobisoxazole cruciforms as emitters for developing high-performance deep blue OLEDs.
\end{abstract}

Received 3rd November 2015 Accepted 19th January 2016

DOI: $10.1039 / c 5 t c 03622 d$

www.rsc.org/MaterialsC of conjugated polymers in organic solar cells (OSC)s, organic light emitting diodes (OLED)s, and organic field effect transistors can vary widely. In contrast, mono-disperse conjugated molecules possess well-defined molecular structures, and can be consistently synthesized with high purity. ${ }^{4}$ Initially, studies on these molecules focused on simple systems comprising one building block. ${ }^{5}$ In recent years, combining alternating electron donating (D) and electron accepting (A) moieties within the same (macro)molecule has emerged as an effective way to manipulate the position of its highest occupied molecular orbital (HOMO) and lowest unoccupied molecular orbital (LUMO). To date, a number of different donor-acceptor molecules have been synthesized and OSCs using small molecules as the active layer have recently reached record-breaking efficiencies above 9.0\%. ${ }^{6}$ Small molecules are the materials of choice in commercial OLEDs due to their superior efficiency and color purity in comparison to polymers. ${ }^{7}$ However, until recently, small molecule OLEDs (SMOLEDs), have suffered from limited solubility. ${ }^{8}$ Therefore there is a need for the development of new materials that can be solution-processed.

While the donor-acceptor approach enables the tuning of the materials' frontier molecular orbitals (FMO)s, due to the extensive delocalization of electrons within conjugated systems most chemical modifications impact the position of both the HOMO and LUMO levels. One tactic to circumvent this phenomenon is through the synthesis of two-dimensional crossconjugated molecules comprising two perpendicular $\pi$-conjugated linear units that intersect via a central aromatic core. As a result 
of the spatial segregation of the FMOs in these "cruciforms" it is possible to semi-independently alter the LUMO or the HOMO level through judicious selection and strategic positioning of substituents around the central molecule. ${ }^{9}$ Benzo[1,2- $\left.d: 4,5-d^{\prime}\right]$ bisoxazole (BBO)based cruciforms are unique since one of the conjugation pathways encompasses the two oxazole rings whereas the other goes through the central benzene ring. ${ }^{10}$ Recently, the Miljanic group reported the use of BBO cruciform as fluorescent sensors. ${ }^{10 e-h}$ Their work, along with our own synthetic and theoretical studies on cross-conjugated BBOs, ${ }^{10 c, 11}$ demonstrates that in the case of donor-acceptor systems, the HOMO level can be localized along perpendicular axis $(4,8-)$ whereas the LUMO level is localized along horizontal axis (2,6-). Thus, selective tuning of the FMOs is possible, although to date has not been widely utilized for developing organic electronics.

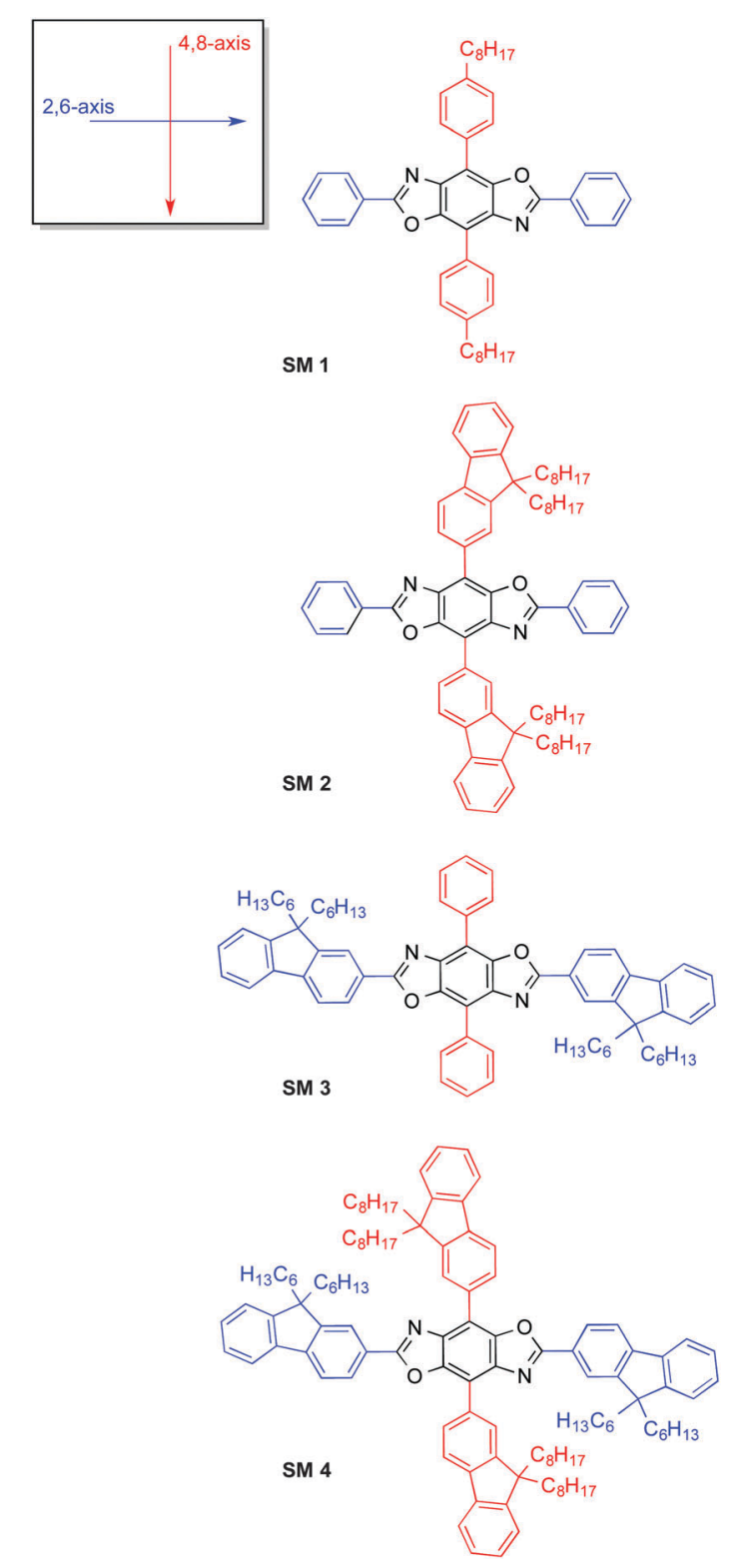

Chart 1 Structures of molecules synthesized in this work.
In this work we demonstrate, for the first time the utility of BBO cruciforms as a tunable platform for the development of organic semiconductors, Chart 1. Based on theoretical calculations, we synthesized four novel cross-conjugated molecules composed of the BBO core and different arrangements of phenyl- and fluorenyl-substitutents. We targeted the synthesis of deep blue light-emitting materials, as they are essential components in solidstate light based on white OLEDs. ${ }^{12}$ These wide band gap materials can also be used as hosts for green and red emitters to achieve the primary color required for displays. ${ }^{13}$ Unfortunately, the requisite wide band gap and corresponding reduction in emission quantum yield make it very challenging to attain efficient deep blue emission. ${ }^{13 b, 14}$ Thus we envisioned that a small molecule with tunable FMOs could best achieve these goals.

\section{Results and discussion}

\section{Synthesis and characterization}

The synthetic approach for the 2,4,6,8-tetraarylBBOs is shown in Scheme 1. All of the molecules were prepared using a simple two step approach: a condensation reaction to prepare the 2,6-diaryl-4,8-dibromoBBOs and then a cross-coupling to extend conjugation across the central benzene ring. This approach allows for the synthesis of multiple BBOs from common intermediates. The condensation reaction of 3,6-diamino-2,5-dibromohydroquinone (1) with benzoyl chloride or 9,9-dihexyl-9H-fluorene2-carbonyl chloride (5), afforded compounds 2 and $\mathbf{6}$ in yields of $23 \%$ and $15 \%$, respectively. Although the yields for these steps were low, we were able to obtain adequate amounts of material for the next step. The cross-coupling of 2 with 4-octylphenylboronic acid (3) or 2-(9,9-dioctyl-9H-fluoren-2-yl)-4,4,5,5-tetramethyl-1,3,2dioxaborolane (4) yielded SM 1 and SM 2 in yields of $60 \%$ and $75 \%$, respectively. The cross coupling of 6 with phenylboronic acid or 4 afforded SM 3 and SM 4 in 40\% and 62\%, yield respectively. Purification of these compounds was easily accomplished by first passing through a short silica gel plug to remove non-polar and residual catalyst followed by precipitation from methanol. Lastly, the small molecules purified by a subsequent column and precipitation. The cruciforms were soluble in chlorinated solvents and characterized by NMR spectroscopy, and high-resolution mass spectrometry. The thermal properties of the materials were studied using differential scanning calorimetry (DSC) under nitrogen and thermogravimetric analysis (TGA) in air with a heating rate of $20{ }^{\circ} \mathrm{C}$. All the BBOs showed excellent thermal stability under ambient conditions with 5\% weight loss temperatures of 368 , 357, 390 and $409{ }^{\circ}$ C for SM 1, SM 2, SM 3 and SM 4 respectively (Fig. S2, ESI $\dagger$ ). DSC did not reveal any transitions pertaining to melting or crystallization before the decomposition temperature. Thus, the heat generated during device operation should not alter the film morphology of these materials.

\section{Optical and electrochemical properties}

The solution UV-Vis absorption and photoluminescence spectra of the BBOs are shown in Fig. S4 (ESI $\dagger$ ) and Fig. 1, respectively. The data is summarized in Table 1. All of the BBOs exhibited 


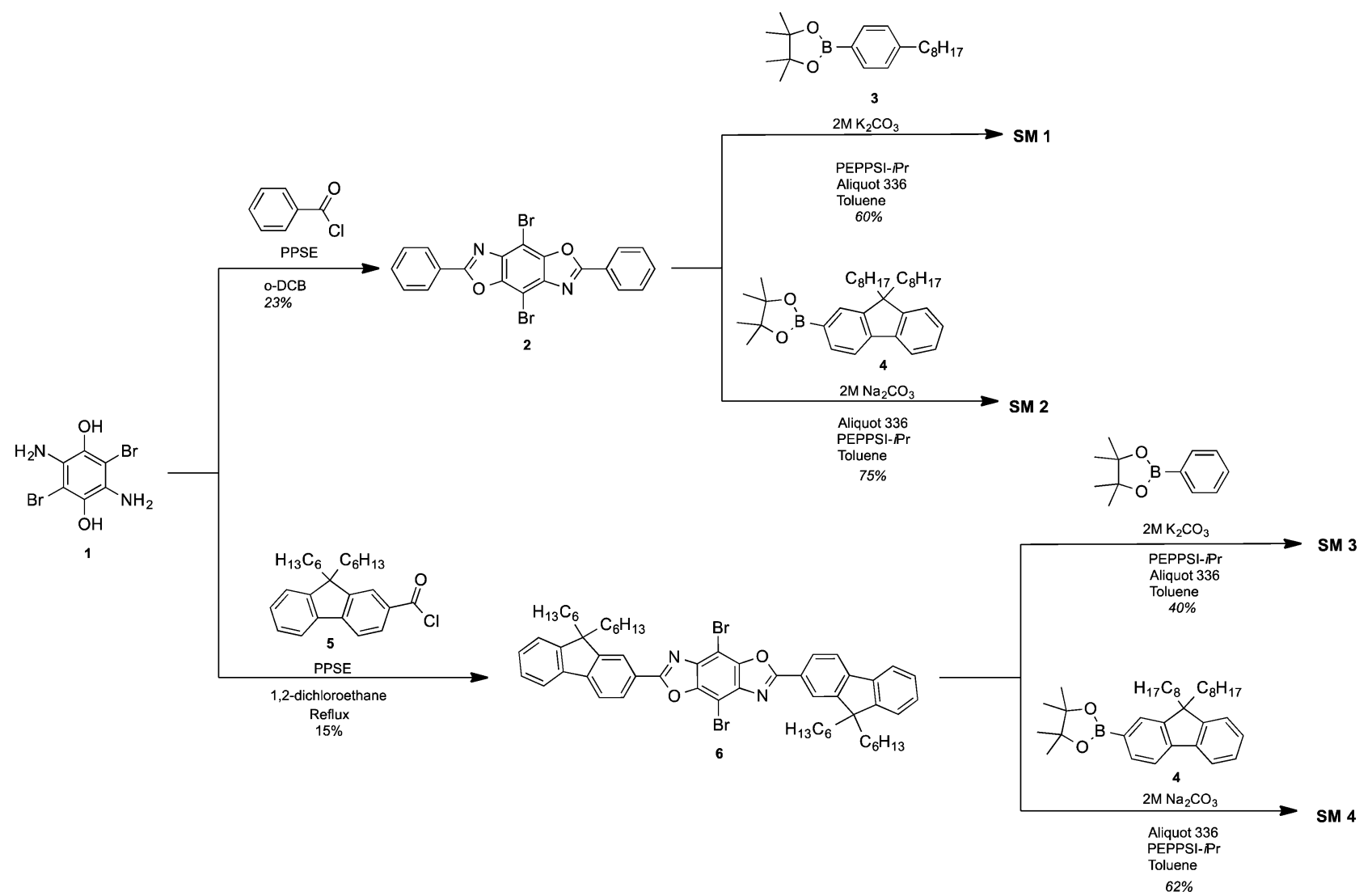

Scheme 1 Synthesis of BBO cruciform.

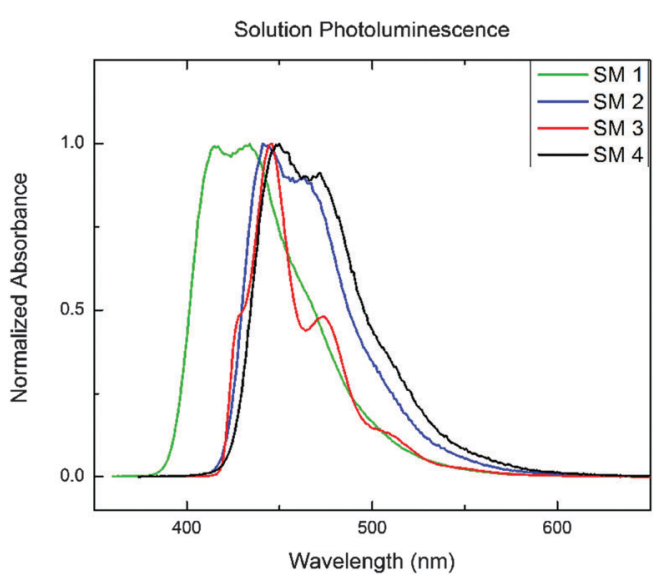

Fig. 1 Photoluminescence spectra of SM 1-SM 4. strong blue-emission in solution (433-450 nm). SM 1, SM 2 and SM 4 all had broad, featureless peaks, whereas SM 3 had a fairly shifted shoulder. The fluorescence quantum yields in solution as SM 1 was the lowest, SM 4 the highest and the constitutional isomers, SM 2 and SM 3 were identical. The electrochemical properties of the BBOs were investigated by cyclic voltammetry (CV) using a platinum working electrode in acetonitrile, with $0.1 \mathrm{M} \mathrm{Bu} \mathrm{NPF}_{6}$ as the electrolyte and an $\mathrm{Ag} / \mathrm{Ag}^{+}$reference electrode. The onsets were referenced to $\mathrm{Fc} / \mathrm{Fc}^{+}$and the results are summarized in Table 1 . All of the materials exhibited measurable and reproducible oxidation and reduction processes. The HOMO values of the BBOs were similar, ${ }^{15}$ ranging from -6.0 to $-6.2 \mathrm{eV}$, whereas the LUMO levels ranged from -2.7 to $-3.7 \mathrm{eV}$. SM 1, with phenyl substituents in all four positions, had the deepest HOMO level $(-6.2 \mathrm{eV})$, and the highest-lying LUMO level $(-2.7 \mathrm{eV})$, resulting in the widest band gap. For the mixed systems possessing both fluorenyl and phenyl groups

Table 1 Optical and electronic properties for SM 1-SM 4

\begin{tabular}{llllllll}
\hline BBO & Abs $\lambda_{\text {max }}^{\text {film }}(\mathrm{nm})$ & $\mathrm{PL} \lambda_{\max }^{\text {film }}(\mathrm{nm})$ & $\Phi_{\mathrm{PL}}$ & $E_{\mathrm{g}}^{\mathrm{opt} a}(\mathrm{eV})$ & $\mathrm{HOMO}^{b}(\mathrm{eV})$ & $\mathrm{LUMO}^{c}(\mathrm{eV})$ \\
\hline SM 1 & 355 & 433 & 0.34 & 3.1 & -6.2 & -2.7 & $E_{\mathrm{g}}^{\mathrm{EC} d}(\mathrm{eV})$ \\
SM 2 & 364 & 441 & 0.51 & 2.9 & -5.7 & -3.5 \\
SM 3 & 386 & 444 & 0.51 & 2.9 & -6.0 & -3.7 \\
SM 4 & 395 & 450 & 0.71 & 2.8 & -6.0 & -3.5 & 2.3 \\
\hline
\end{tabular}

${ }^{a}$ Estimated from the absorption onset of the film. ${ }^{b} \mathrm{HOMO}=-\left(E_{\mathrm{Onset}}^{\mathrm{ox}}+4.8\right) \mathrm{eV} \cdot{ }^{c} \mathrm{LUMO}=-\left(E_{\mathrm{onset}}^{\mathrm{red}}+4.8\right) \mathrm{eV} .{ }^{d} E_{\mathrm{g}}^{\mathrm{EC}}=\mathrm{LUMO}-\mathrm{HOMO}$. 


$$
\text { HOMO }
$$

SM 1

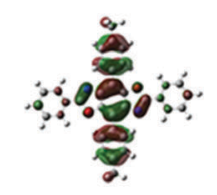

SM 2

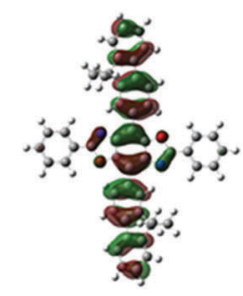

SM 3

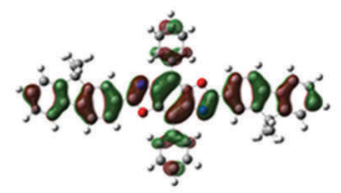

SM 4

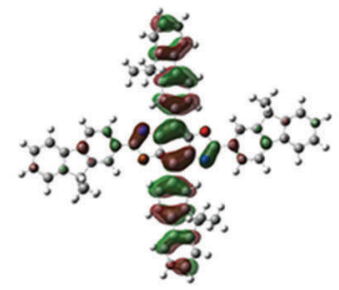

Fig. 2 Frontier orbitals for SM 1-SM 4.

(SM 2 and SM 3) the band gaps were both $2.9 \mathrm{eV}$. Since both the phenyl and fluorenyl substitutents are weakly electron-donating the difference in the energy levels in these system is likely a result of the difference in conjugation length. ${ }^{10 c}$ This conclusion is verified by the band gap trend SM $1>$ SM $2=$ SM $3>$ SM 4. The influence of substitutent selection, and location is further revealed in frontier orbital diagrams above (Fig. 2).

The electrochemical properties of the BBOs were investigated by cyclic voltammetry (CV) using a platinum working electrode in acetonitrile, with $0.1 \mathrm{M} \mathrm{Bu}_{4} \mathrm{NPF}_{6}$ as the electrolyte and an $\mathrm{Ag} / \mathrm{Ag}^{+}$reference electrode. The onsets for the $\mathrm{BBOs}$ ranged from 0.34-0.71. An increase in quantum yield was seen upon the addition of fluorene to the BBO core, narrow peak-width at halfmax with a bathochromically-had.

\section{Theoretical calculations}

To further evaluate the structure-property relationships within these cross-conjugated BBOs, time-dependent density functional theory was performed. Initially, all four materials were optimized and their excited states generated using B3LYP/6-31G*. Based on previous benchmarking studies, ${ }^{16}$ it was determined that the MPW1PBE level with an SV basis set combined with the polarizable conductor calculation model (CPCM) ${ }^{17}$ mimicking the $\mathrm{CHCl}_{3}$ solvation effects provided the best correlation to experiment. Both sets of computational results were compared and contrasted to experimental CV and UV-Vis data and are summarized in Table 2 where the absolute HOMO and the band gap measurements was $0.2 \mathrm{eV}$ leading to $3.5 \%$ and $6.9 \%$ errors, respectively. Conversely, the LUMO comparison suffered from much larger absolute and percent errors, which was expected based on the error in the $\mathrm{CV}$ as well as given the limitations inherent in DFT computations. ${ }^{15}$

The HOMO levels predicted by theory demonstrate the impact substituent identify plays along the 4,8-axis. Phenyl substituents (SM 1 and SM 3) lower the HOMO to $-6.1 \mathrm{eV}$, whereas fluorenyl rings raise the HOMO to $-5.9 \mathrm{eV}$ (SM 2 and SM 4). The band gaps appear to be influenced by conjugation length as well as the extent of delocalization of electron density as shown in the frontier orbitals displayed in Fig. 3. There was localization along the donor axis for the HOMOs with the exception of SM 3. Both SM 2 and SM 4 demonstrated more localization along the acceptor axis than SM 3 or SM 1. These two trends along with conjugation length led to both lower band gaps and may play a part in the improved performance of SM 2 and SM 4 in OLEDs relative to SM 1 and SM 3. The importance of aryl ring identity was further examined by measuring the torsion angle produced between the 4,8-axis and the plane possessing the 2,6-axis, Fig. 3. The larger the torsion suppress the intermolecular aggregation between the BBO cores enhancing their efficiency in OLEDs. ${ }^{18}$ The ranking in order of increasing dihedral angle was: SM $4<$ SM $2<$ SM $1<$ SM 3. As discussed below, SM 1 could not be fabricated into a working device with the CBP host material. SM 3 indicated unfavourable electron distribution in the frontier orbitals, thus SM 2 and SM 4, are the most viable candidates for use in OLEDs. Based on the torsion angle data, theory predicts that a SM 2 should produce better device than SM 4, and this is corroborated by the data below.

\section{Organic light emitting diodes}

The EL of the cruciforms was evaluated in host-guest OLEDs using 4,4'-bis(9-carbazolyl)-biphenyl (CBP) as the small molecule host. The configuration for these devices was ITO/PEDOT:PSS $(60 \mathrm{~nm}) /$ CBP:BBO/BPhen $(40 \mathrm{~nm}) / \mathrm{LiF}(1 \mathrm{~nm}) / \mathrm{Al}(100 \mathrm{~nm})$. The energy level

Table 2 Theoretical computations compared to the experimental optical and electronic measurements for SM 1-SM 4

\begin{tabular}{|c|c|c|c|c|c|c|c|c|c|}
\hline \multirow[b]{2}{*}{ BBO } & \multicolumn{3}{|c|}{ HOMO (abs diff. from exp't) in eV } & \multicolumn{3}{|c|}{ LUMO (abs diff. from exp't) in eV } & \multicolumn{3}{|c|}{$E_{\mathrm{g}}$ (abs diff. from exp't) in eV } \\
\hline & Exp't & B3LYP/6-31G* & MPW1PBE/SV-CHCl ${ }_{3}$ & Exp’t & B3LYP/6-31G* & MPW1PBE/SV-CHCl 3 & Exp't & B3LYP/6-31G* & $\mathrm{MPW} 1 \mathrm{PBE} / \mathrm{SV}-\mathrm{CHCl}_{3}$ \\
\hline SM 1 & -6.2 & $-5.3(0.9)$ & $-6.1(0.1)$ & -2.7 & $-1.9(0.8)$ & $-2.4(0.3)$ & 3.1 & $3.2(0.1)$ & $3.2(0.1)$ \\
\hline SM 2 & -5.7 & $-5.2(0.5)$ & $-5.9(0.2)$ & -3.5 & $-1.9(1.6)$ & $-2.5(1.0)$ & 2.9 & $2.9(0.0)$ & $2.9(0.0)$ \\
\hline SM 3 & -6.0 & $-5.3(0.7)$ & $-6.1(0.1)$ & -3.7 & $-2.0(1.7)$ & $-2.5(1.2)$ & 2.9 & $3.0(0.1)$ & $3.1(0.2)$ \\
\hline SM 4 & -6.0 & $-5.1(0.9)$ & $-5.9(0.1)$ & -3.5 & $-2.0(1.5)$ & $-2.5(1.0)$ & 2.8 & $2.8(0.0)$ & $2.9(0.1)$ \\
\hline
\end{tabular}



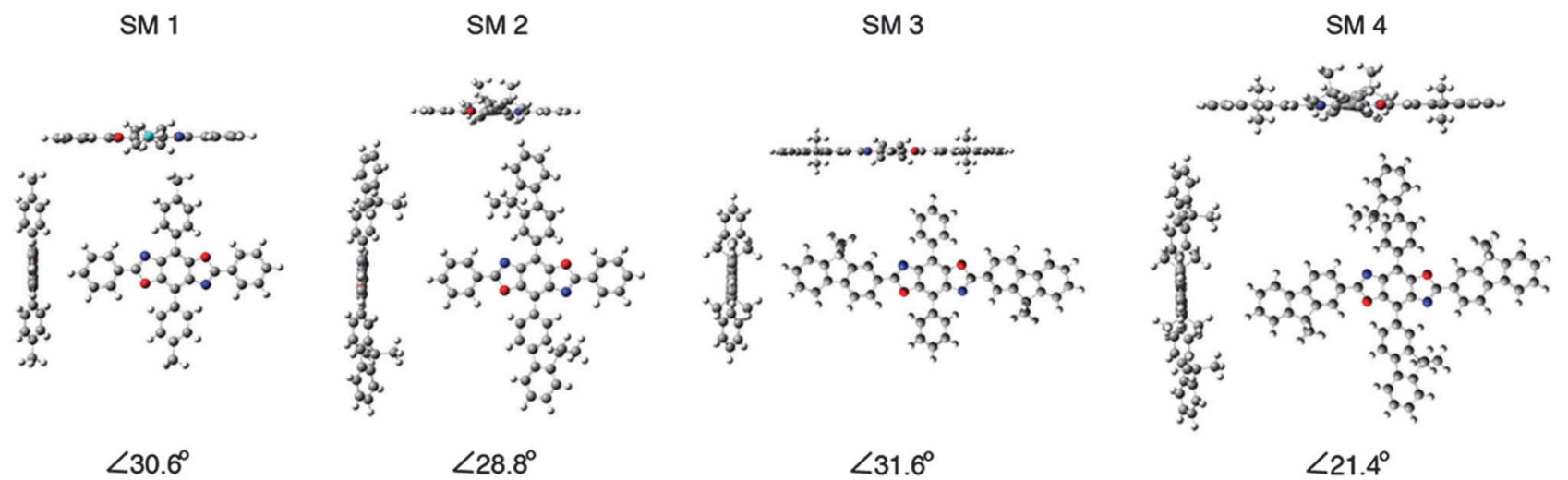

Fig. 3 Optimized geometries for SM 1-SM 4.

diagrams and the EL spectra of devices comprising the CBP host and $10 \%$ of the guest (SM 1, SM 2 or SM 3) are shown in Fig. 4 and 5a, respectively. Due to the poor performance of the device from SM 1, the EL spectra could not be obtained. This result is not surprising as both the HOMO and LUMO levels for SM 1 are outside those for the CBP host. The emission maximum of SM 2 is red-shifted $11 \mathrm{~nm}$ from SM 3, demonstrating the influence of the direction of the extended conjugation axis. Similarly, the emission maximum of SM $\mathbf{4}$ was red-shifted $22 \mathrm{~nm}$ relative to SM 3 as a result of extended conjugation. The EL spectrum for SM 3 is similar in width and shape to its emission spectrum, but the peak widths at half-maximum for the EL spectra of SM $\mathbf{2}$ and SM $\mathbf{4}$ are considerably narrower than their emission spectra. The normalized electroluminescent spectra of devices with different weight\% concentrations of the BBO's in the CBP host are shown below in Fig. 5. The key performance parameters of these OLEDs are summarized in Table 3. It can be seen from these plots that the host is quenched upon the addition of $\sim 5 \%$ of the guest molecule, indicating that there is efficient energy transfer from the host to the emitters. Interestingly, the best devices were obtained when $10 \mathrm{wt} \%$ of the guest was used. These results are also an

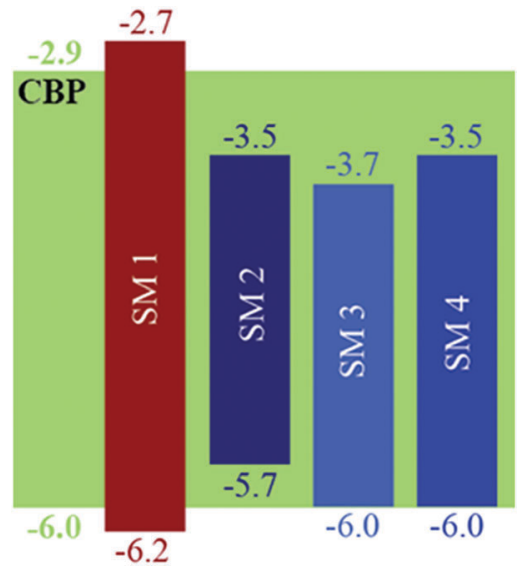

Fig. 4 Energy level diagram of SM 1-SM 4 and CBP host. improvement from our previous work where BBO polymer: CBP host systems were studied, as emission was not observed with $10 \%$ doping levels. ${ }^{19}$ This relatively high concentration of the guest suggests that excellent guest-host mixing is occurring in this system, as OLEDs based on neat films of the cruciforms exhibited strong concentration quenching. Such quenching is often a result of strong $\pi$-stacking within conjugated systems. Based on the optimized geometries in Fig. 3, we could surmise that SM 2, was the least planar and thus least susceptible to intermolecular aggregation. Indeed, SM 2, gave the best performance of all the molecules with an efficiency of $\sim 2 \mathrm{~cd} \mathrm{~A}^{-1}$ and a peak emission at $443 \mathrm{~nm}$, when doped into the host at $10 \mathrm{wt} \%$. The CIE 1931 coordinates for SM 2 of $x=0.16, y=0.05$, were well into the deep blue region. Interestingly, switching the conjugation axis had a detrimental impact on the OLED performance as SM 3 had the lowest efficiency, luminescence and EQE regardless of the doping level. This may be a result of the difference in the distribution of the electron density within the system as seen in the frontier orbitals, Fig. 3. Another contributing factor is that the deep HOMO level of SM 3 is close to that of the CBP guest. However, since SM $\mathbf{4}$ has the same HOMO level as SM $\mathbf{3}$ and performed significantly better, energy level misalignment is not likely the cause of the poor performance. In the future we will utilize a wider band gap host to further evaluate this phenomena. The device made using $2 \%$ of SM 4 gave the second best performance an efficiency of $\sim 1.9 \mathrm{~cd} \mathrm{~A}^{-1}$, with peak emission at $443 \mathrm{~nm}$, and CIE 1931 coordinates $x=0.15, y=0.09$, which are close to the National Television System Committee (NTSC) blue color standard $(0.14,0.08) .{ }^{18,20}$ These are very promising materials considering that the photopic factor is only 0.023 at $\sim 445 \mathrm{~nm}$. Furthermore, considering that the PL quantum yield is only 0.5 , the maximum internal quantum efficiency is only $\sim 0.25 \times 0.5=0.125$ (yield of singlets times PL quantum yield), and the EQE is about $\sim 0.2 \times 0.125=0.025=2.5 \%$, since the outcoupling factor in these conventional bottom-emitting-through-theglass OLEDs with ITO is $<20 \%$. Hence, based on the $\Phi_{\mathrm{PL}}$ of the emitting materials, the EQE of the deep blue fluorescent OLEDs based on SM 2 and SM 4 are close to the maximal possible values. 

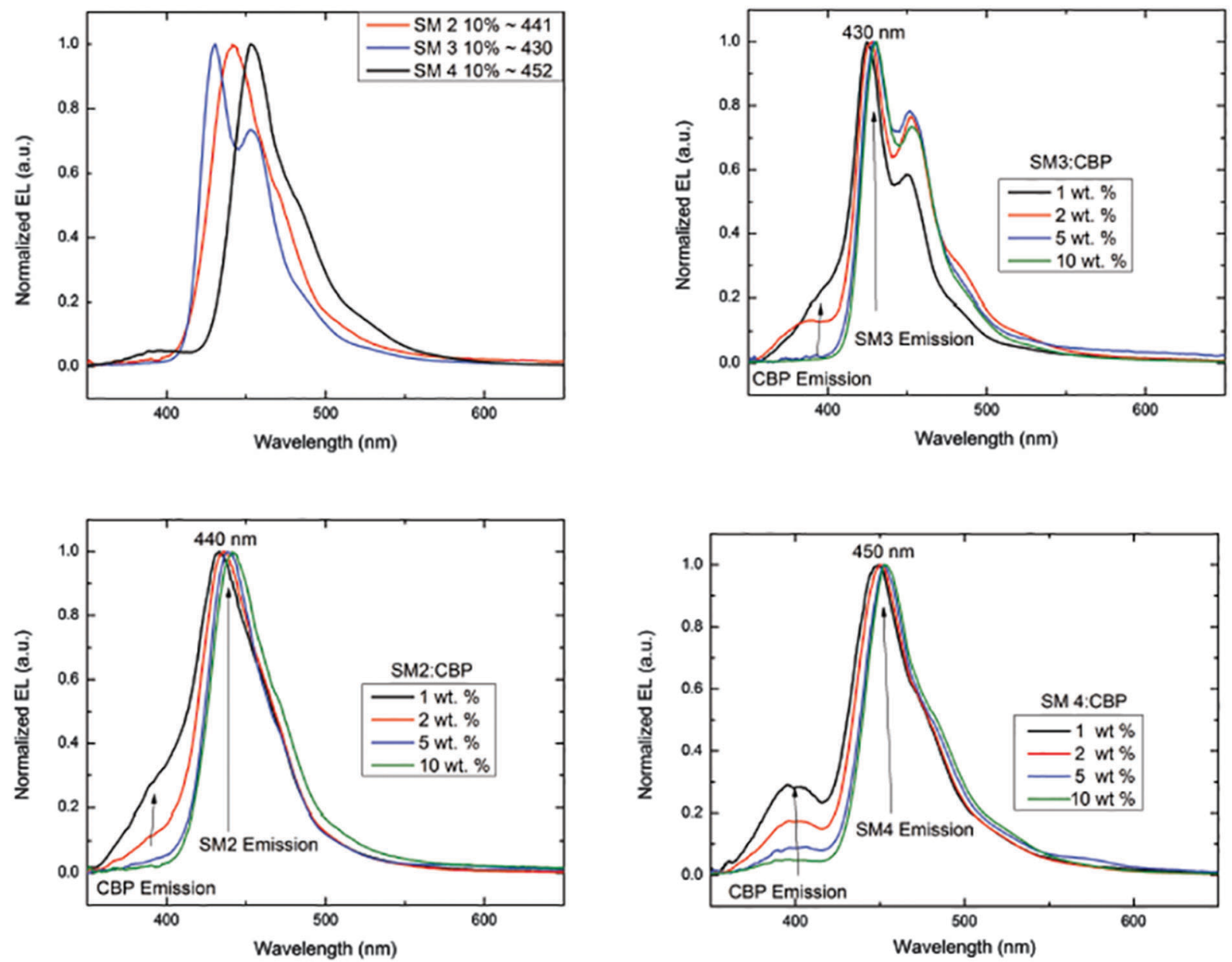

Fig. 5 Electroluminescence spectra of SM 2-SM 4 (top left), and EL emission at various concentrations as noted.

Table 3 Device characteristics of SMOLEDs based on BBO cruciforms

\begin{tabular}{|c|c|c|c|c|c|c|c|c|}
\hline Dopant & Device wt\% & $V_{\text {on }}[\mathrm{V}]$ & $\begin{array}{l}\text { Drive } \\
\text { voltage }[\mathrm{V}]\end{array}$ & $\begin{array}{l}\text { Current density } \\
J,\left[\mathrm{~mA} \mathrm{~cm}^{-2}\right]\end{array}$ & $\begin{array}{l}\text { Brightness } \\
{\left[\mathrm{cd} \mathrm{m}^{-2}\right]}\end{array}$ & $\begin{array}{l}\text { Efficiency }\left[\mathrm{cd} \mathrm{A}^{-1},\right. \\
\left.\operatorname{lm~} \mathrm{W}^{-1}(\% \mathrm{EQE})\right]\end{array}$ & $\lambda_{\max }^{\mathrm{EL}}[\mathrm{nm}]$ & CIE $1931\left[x, y, y^{\prime}\right]$ \\
\hline \multirow{3}{*}{ SM 2} & 1.0 & 4.2 & 6.2 & 392 & 1155 & $0.93,0.61,1.82$ & 433,400 & $0.16,0.05,0.79$ \\
\hline & 5.0 & 4.4 & 6.6 & 489 & 1141 & $0.71,0.45,1.08$ & 439,400 & $0.16,0.06,0.78$ \\
\hline & 10.0 & 4.0 & 7.0 & 336 & 1051 & $2.05,1.40,2.95$ & 443,400 & $0.16,0.05,0.79$ \\
\hline \multirow{2}{*}{ SM 3} & 1.0 & 5.0 & 8.0 & 365 & 275 & $0.33,0.20,0.60$ & 425,400 & $0.17,0.06,0.78$ \\
\hline & 10.0 & 4.2 & 8.2 & 583 & 779 & $0.50,0.33,0.76$ & 430,400 & $0.16,0.06,0.78$ \\
\hline \multirow[t]{4}{*}{ SM 4} & 1.0 & 4.0 & 6.3 & 360 & 1649 & $1.23,0.77,1.55$ & 450,400 & $0.15,0.09,0.76$ \\
\hline & 2.0 & 4.0 & 6.4 & 350 & 1568 & $1.93,1.24,2.22$ & 450,400 & $0.15,0.09,0.76$ \\
\hline & 5.0 & 3.9 & 6.4 & 404 & 1412 & $1.09,0.67,1.01$ & 450,400 & $0.16,0.11,0.73$ \\
\hline & 10.0 & 3.6 & 6.9 & 606 & 1159 & $1.29,0.97,1.20$ & 450,400 & $0.15,0.11,0.74$ \\
\hline
\end{tabular}

\section{Conclusions}

In summary, we have synthesized four cross-conjugated, blue light emitting materials based on benzo[1,2-d:4,5- $\left.d^{\prime}\right]$ bisoxazole. The HOMO and LUMO levels of these materials were readily tuned via substitution pattern to afford materials with wide-band gaps. Structure-property studies reveal that such modifications not only impact the energy levels and band gaps, but they also affect the electron density and planarity within the systems. As a result, the performance of the OLEDs varied depending upon structure. Non-optimized OLEDs achieved EQE approaching $3 \%$ and efficiency of $\sim 2 \mathrm{~cd} \mathrm{~A}^{-1}$ with a relatively narrow EL band peaking at $443 \mathrm{~nm}$ and a $\mathrm{CIE}_{y} \leq 0.05$. Collectively these results indicate that the synthesis of $\mathrm{BBO}$ cruciforms is a 
promising strategy for the design of efficient deep-blue OLEDs. Future work is ongoing in our labs on OLED optimization and synthesis of new materials. These will be reported in due course.

\section{Experimental}

\section{Materials and measurements}

Br-DAHQ (1), ${ }^{21}$ polyphosphoric acid, silyl ester (PPSE), ${ }^{22}$ were synthesized according to literature procedures. THF and toluene were dried using an Innovative Technologies solvent purification system. All other chemical reagents were purchased from commercial sources and used without further purification unless otherwise noted. Nuclear magnetic resonance (NMR) experiments were carried out in $\mathrm{CDCl}_{3}$ at $600 \mathrm{MHz}\left({ }^{1} \mathrm{H}\right)$ AND $800 \mathrm{MHz}\left({ }^{13} \mathrm{C}\right)$. In all spectra, chemical shifts are given in $\delta$ relative to tetramethylsilane. Coupling constants are reported in hertz $(\mathrm{Hz})$. High-resolution mass spectra were recorded on a double-focusing magnetic sector mass spectrometer using ESI or APCI. Melting points were obtained on a melting point apparatus with $260{ }^{\circ} \mathrm{C}$ upper limit and are uncorrected. All UV-Vis and fluorescence spectroscopy were obtained using $\mathrm{CHCl}_{3}$ solutions unless otherwise noted. Relative solution fluorescence quantum yields were obtained using 9,10-diphenylanthracene $\left(\Phi_{\mathrm{PL}}=\right.$ 0.885 in ethanol) $)^{23}$ as a standard with excitation at $372 \mathrm{~nm}$.

4,8-Dibromo-2,6-diphenylbenzo[1,2-d:4,5-d']bis(oxazole) (2). In a dried round-bottomed flask (RBF), a fresh prepared solution of poly-phosphosilylether (PPSE) $(5 \mathrm{~g}$ in $20 \mathrm{~mL}$ of $o$-DCB) and $\mathbf{1}$ was degassed for 20 minutes. Benzoyl chloride was added and the solution was refluxed for 2 days under argon. The solution was allowed to cool to room temperature and crashed into cold methanol $\left(-78{ }^{\circ} \mathrm{C}\right)$. The precipitate was filtered and refluxed in EtOH to remove the red color, resulting in a tan powder. The resulting product was used without further purification. M.P. $>260{ }^{\circ} \mathrm{C}$ Due to the poor solubility of the product, NMR spectra were not obtained.

4,8-Dibromo-2,6-bis(9,9-dihexyl-9H-fluoren-2-yl)benzo[1,2-d:4,5$\boldsymbol{d}^{\prime}$ ] bis(oxazole) (6). In a dried RBF, compound 1 (1.49 g, $5 \mathrm{mmol}$ ) and a fresh prepared solution of poly-phosphosilylether (PPSE) $(2 \mathrm{~g}$ in $20 \mathrm{~mL}$ of DCE) were degassed for 20 minutes and then heated at reflux for 3 days under argon. The solution was allowed to cool to room temperature and quenched with $2 \mathrm{M}$ sodium hydroxide and extracted with methylene chloride. The combined organic layers were washed with $2 \mathrm{M}$ hydrochloric acid, water, brine and dried over sodium sulphate then concentrated. The resulting residue was filtered a small silica gel column with $\mathrm{CH}_{2} \mathrm{Cl}_{2}$ and concentrated in vacuo. The residue was put through a second column with $80 / 20 \mathrm{v} / \mathrm{v}$ hexanes $/ \mathrm{CH}_{2} \mathrm{Cl}_{2}$. The solution was concentrated and recrystallized from ethyl acetate.

M.P. $>260{ }^{\circ} \mathrm{C},{ }^{1} \mathrm{H}$ NMR $\left(400 \mathrm{MHz}, \mathrm{CDCl}_{3}\right) 8.36(2 \mathrm{H}, \mathrm{d}, J=4)$, $8.30(2 \mathrm{H}, \mathrm{s}), 7.86(2 \mathrm{H}, \mathrm{d}, J=8), 7.79(2 \mathrm{H}, \mathrm{dd}, J=4,4), 7.39(6 \mathrm{H}, \mathrm{d}$, $J=4), 2.10(9 \mathrm{H}, \mathrm{m}), 1.06(24 \mathrm{H}, \mathrm{m}), 0.75(12 \mathrm{H}, \mathrm{t}), 0.60(9 \mathrm{H}, \mathrm{m})$; HRMS (ESI) calcd for $\mathrm{C}_{58} \mathrm{H}_{66} \mathrm{~N}_{2} \mathrm{O}_{2} \mathrm{Br}_{2} 981.3564[\mathrm{M}+\mathrm{H}]^{+}$, found 981.3559.

4,8-Bis(4-octylphenyl)-2,6-diphenylbenzo[1,2-d:4,5-d']bis(oxazole) (SM 1). In a dried, 3-necked RBF purged with argon, fitted with an condenser, monomers $2(117.5 \mathrm{mg}, 0.25 \mathrm{mmol})$ and $3(174 \mathrm{mg}$, $0.55 \mathrm{mmol}$ ) and were dissolved in degassed toluene. One drop of Aliquot 336 (surfactant) was added to the reaction flask, followed by sodium carbonate $(6 \mathrm{~mL}, 10 \mathrm{mmol})$. The reaction solution was then deoxygenated for 30 min by pumping argon through the solution. PEPPSI-iPr catalyst $(0.0125 \mathrm{mmol})$ was added and the reaction refluxed for 3 days under argon. The solution was then diluted with toluene and layers separated. Aqueous layer was extracted twice with toluene $(2 \times 20 \mathrm{~mL}$ portions). The combined organic layers were washed with $1 \mathrm{M}$ hydrochloric acid, water, brine and dried with sodium sulfate. After concentration, the residue was purified by column chromatography (gradient hexanes to $50 / 50 \mathrm{v} / \mathrm{v}$ hexanes/dichloromethane). The eluents were concentrated and dissolved in $1.5 \mathrm{~mL}$ of chloroform and crashed out into methanol $\left(-78{ }^{\circ} \mathrm{C}\right)$. The resulting powder was taken through a second column by dry packing to silica, following the same gradient then precipitated into $\mathrm{MeOH}$ to give the product as a white powder ( $87 \mathrm{mg}, 50 \%$ ).

M.P. $242{ }^{\circ} \mathrm{C},{ }^{1} \mathrm{H}$ NMR $\left(600 \mathrm{MHz}, \mathrm{CDCl}_{3}\right) \delta 8.34-8.37(8 \mathrm{H}, \mathrm{m}$, $J=6,6 \mathrm{~Hz}), 7.54(6 \mathrm{H}, \mathrm{d}, J=6 \mathrm{~Hz}), 7.47(4 \mathrm{H}, \mathrm{d}, J=6 \mathrm{~Hz}), 2.75(4 \mathrm{H}$, m), $1.75(4 \mathrm{H}, \mathrm{m}) 1.55(13 \mathrm{H}, \mathrm{s}) 1.45(5 \mathrm{H}, \mathrm{m}), 1.38(4 \mathrm{H}, \mathrm{m}) 1.32$ $(10 \mathrm{H}, \mathrm{m}) 0.90(6 \mathrm{H}, \mathrm{t}) ;{ }^{13} \mathrm{C}\left(400 \mathrm{MHz}, \mathrm{CDCl}_{3}\right) \delta 165.68,152.10$, 152.00, 147.37, 146.04, 140.35, 140.21, 128.91, 127.95, 127.53, $124.95,123.50,122.84,121.61,120.61,91.86,56.03,40.83$, 32.01, 30.12, 24.22, 23.03, 14.46; HRMS (ESI) calcd for $\mathrm{C}_{48} \mathrm{H}_{52} \mathrm{~N}_{2} \mathrm{O}_{2} 689.4102[\mathrm{M}+\mathrm{H}]^{+}$, found 689.4087.

4,8-Bis(9,9-dioctyl-9H-fluoren-2-yl)-2,6-diphenylbenzo[1,2-d:4,5$d^{\prime}$ ]bis(oxazole) (SM 2). SM 2 was synthesized similarly to SM 1 from 2 and 4 . The product was obtained as a bright yellow powder (90 mg, 76\%).

M.P. $149-152{ }^{\circ} \mathrm{C},{ }^{1} \mathrm{H}$ NMR $\left(800 \mathrm{MHz}, \mathrm{CDCl}_{3}\right) \delta 8.50(2 \mathrm{H}, \mathrm{m}$, $J=8,8 \mathrm{~Hz}), 8.45(2 \mathrm{H}, \mathrm{s}), 8.37(4 \mathrm{H}, \mathrm{dd}, J=8,8 \mathrm{~Hz}), 7.99(2 \mathrm{H}, \mathrm{d}$, $J=8 \mathrm{~Hz}), 7.84(4 \mathrm{H}, \mathrm{d}, J=8 \mathrm{~Hz}), 7.75(2 \mathrm{H}, \mathrm{d}, J=8 \mathrm{~Hz}), 7.45(2 \mathrm{H}, \mathrm{d}$, $J=8 \mathrm{~Hz}) 7.42(2 \mathrm{H}, \mathrm{td}, J=8 \mathrm{~Hz}) ; 7.38(2 \mathrm{H}, \mathrm{td}, J=8 \mathrm{~Hz}), 2.15$ $(10 \mathrm{H}, \mathrm{m}), 1.17(18 \mathrm{H}, \mathrm{m}), 0.95(4 \mathrm{H}, \mathrm{m}), 0.82(4 \mathrm{H}, \mathrm{m}), 0.78(14 \mathrm{H}, \mathrm{t}$, $J=8 \mathrm{~Hz}) ;{ }^{13} \mathrm{C}\left(800 \mathrm{MHz}, \mathrm{CDCl}_{3}\right) \delta 163.66,151.51,150.88,146.62$, 141.28, 141.05, 138.60, 131.61, 131.33, 129.29, 129.27, 129.01, $127.80,127.50,127.39,127.00,125.07,125.05,123.15,120.16$, 119.97, 114.99, 55.37, 40.82, 31.92, 31,90, 30.53, 29.54, 29.53, $29,46,24.20,22.73,22.72,14.19,14.18$ HRMS (ESI) calcd for $\mathrm{C}_{78} \mathrm{H}_{92} \mathrm{~N}_{2} \mathrm{O}_{2}$ 1089.7232 [M+ H] $]^{+}$, found 1089.7228.

2,6-Bis(9,9-dihexyl-9H-fluoren-2-yl)-4,8-diphenylbenzo[1,2-d:4,5$d^{\prime}$ ]bis(oxazole) (SM 3). SM 3 was synthesized similarly to SM 1 from $\mathbf{6}$ and phenyl boronic ester. The resulting residue was purified by column chromatography (gradient hexanes to $50 / 50 \mathrm{v} / \mathrm{v}$ hexanes/ dichloromethane). The product was obtained as light-yellow flakes (97 mg, 40\%).

M.P. $214{ }^{\circ} \mathrm{C},{ }^{1} \mathrm{H}$ NMR $\left(600 \mathrm{MHz}, \mathrm{CDCl}_{3}\right) \delta 8.49(2 \mathrm{H}, \mathrm{dd}$, $J=6 \mathrm{~Hz}), 8.44(2 \mathrm{H}, \mathrm{s}), 8.37(4 \mathrm{H}, \mathrm{m}), 7.98(2 \mathrm{H}, \mathrm{d}, J=6 \mathrm{~Hz}), 7.82$ $(2 \mathrm{H}, \mathrm{d}), 7.56(6 \mathrm{H}, \mathrm{m}), 6.14(6 \mathrm{H}, \mathrm{m}), 2.14(9 \mathrm{H}, \mathrm{ddq}), 1.13$ $(45 \mathrm{H}, \mathrm{dd}), 0.76(14 \mathrm{H}, \mathrm{t}) ;{ }^{13} \mathrm{C}\left(800 \mathrm{MHz}, \mathrm{CDCl}_{3}\right) \delta 164.81$, 151.88, 151.68, 146.74, 144.99, 140.37, 138.84, 133.03, 130.69, 130.67, 130.66, 129.01, 128.61, 128.44, 127.34, 127.29, 125.89, 123.30, 122.38, 120.31, 114.45, 55.72, 40.60, 31.85, 29.95, 24.07, 14.31; HRMS (ESI) calcd for $\mathrm{C}_{70} \mathrm{H}_{76} \mathrm{~N}_{2} \mathrm{O}_{2} 977.598[\mathrm{M}+\mathrm{H}]^{+}$, found 977.5982 . 
2,6-Bis(9,9-dihexyl-9H-fluoren-2-yl)-4,8-bis(9,9-dioctyl-9H-fluoren2-yl)benzo[1,2-d:4,5- $\left.d^{\prime}\right]$ bis(oxazole) (SM 4). SM 4 was synthesized similarly to SM 1 from $\mathbf{4}$ and $\mathbf{6}$. The product was obtained as yellow crystals (208 mg, 52\%).

M.P. ${ }^{144-146}{ }^{\circ} \mathrm{C},{ }^{1} \mathrm{H}$ NMR $\left(600 \mathrm{MHz}, \mathrm{CDCl}_{3}\right){ }^{1} \mathrm{H}$ NMR $\left(600 \mathrm{MHz}, \mathrm{CDCl}_{3}\right) 8.62(2 \mathrm{H}, \mathrm{d}, J=6 \mathrm{~Hz}), 8.55(2 \mathrm{H}, \mathrm{s}), 8.32$ $(4 \mathrm{H}, \mathrm{m}, J=12 \mathrm{~Hz}), 7.99(2 \mathrm{H}, \mathrm{d}, J=6 \mathrm{~Hz}), 7.81(4 \mathrm{H}, \mathrm{t}, J=6 \mathrm{~Hz})$, $7.73(2 \mathrm{H}, \mathrm{dd}), 7.40(2 \mathrm{H}, \mathrm{d}, J=6 \mathrm{~Hz}) 7.33(10 \mathrm{H}, \mathrm{m}, J=6,6 \mathrm{~Hz}) ;{ }^{13} \mathrm{C}$ $\left(800 \mathrm{MHz}, \mathrm{CDCl}_{3}\right) \delta 164.44,151.76,151.53,151.11,146.87$, 144.99, 141.48, 141.35, 140.45, 138.90, 131.83, 129.53, 129.50, 128.47, 127.56, 127.32, 127.15, 126.16, 125.05, 125.02, 123.37, $123.32,122.22,120.69,120.34,120.31,120.18,114.70,55.81$, $55.66, \quad 41.13,40.83,32.11,31.89, \quad 30.76,30.04, \quad 29.70$, 29.68, 24.39, 24.10, 22.90, 14.33,14.31; HRMS (ESI) calcd for $\mathrm{C}_{116} \mathrm{H}_{148} \mathrm{~N}_{2} \mathrm{O}_{2}$ 1602.1614 [M+ H] $]^{+}$, found 1602.1583.

\section{OLED fabrication and characterization}

OLEDs were fabricated on nominally $10-20 \mathrm{ohms} \mathrm{sq}{ }^{-1}, 120$ $160 \mathrm{~nm}$ thick ITO-coated glass substrates (Colorado Concept Coatings). The substrates were first cleaned with a detergent and organic solvents and then treated in a UV/ozone oven to increase the work function of the ITO and hence facilitate hole injection, as described elsewhere. A $60 \mathrm{~nm}$ poly(3,4-ethylenedioxythiophene):polystyrene sulfonate (PEDOT:PSS) layer was spin-coated onto the ITO and then baked in air at $120^{\circ} \mathrm{C}$ for $1 \mathrm{~h}$ and next in an argon-filled glove box at $120{ }^{\circ} \mathrm{C}$ for another $30 \mathrm{~min}$. Blends of 4,4'-bis(9-carbazolyl)-biphenyl (CBP) and 1, 2, 5 and $10 \mathrm{wt} \%$ of SM 1-SM 4 in chlorobenzene solutions were spin-coated on top of the PEDOT:PSS layer in the argon-filled glovebox. The combined concentration of the CBP and guest material was kept constant at $9 \mathrm{mg} \mathrm{mL}{ }^{-1}$. The solution was spin-coated at $4000 \mathrm{rpm}$ for $60 \mathrm{~s}$. The fabricated structure was then annealed at $60{ }^{\circ} \mathrm{C}$ for $30 \mathrm{~min}$. Following this annealing step, the samples were transferred to a thermal evaporator within the glovebox, and the 4,7-diphenyl-1,10-phenanthroline (BPhen), LiF, and Al layers were deposited sequentially by thermal evaporation at a base pressure of $\sim 1 \times 10^{-6}$ Torr. The OLEDs were characterized by monitoring their electroluminescence (EL) spectra and brightness as a function of the applied voltage, and the luminous and power efficiencies were determined.

\section{Computational details}

All of the calculations on these oligomers studied in this work were studied using the Gaussian $03 \mathrm{~W}$ with the GaussView 4 GUI interface program package. All electronic ground states geometries were optimized using density functional theory (DFT) employing an SVP functional and a $6-31 \mathrm{G}^{*}$ basis set. Excited states were generated through time dependent density functional theory (TD-DFT) applied to the optimized ground state for each oligomer. The HOMO, LUMO, band gap, first ten excited states, and UV-Vis simulations were generated from these excited computations. All computations were performed using Gaussian 09 using the Marcy cluster provided by the MERCURY consortium.

\section{Acknowledgements}

We would like to thank Dr Sarah Cady, Dr Kamel Harrata and Mr Steven Veysey of Iowa State University (ISU) Chemical Instrumentation Facility for compound analysis. We thank Eeshita Manna for technical assistance. We also thank the National Science Foundation (CHE-1413173) for financial support of this work. RK and JS were partially supported by Basic Energy Sciences, Division of Materials Science and Engineering, USDOE. Ames Laboratory is operated by Iowa State University for the US Department of Energy (USDOE) under Contract No. DE-AC 02-07CH11358. Computational resources were provided in part by the MERCURY consortium (http://mercuryconsortium.org/) under NSF grants CHE-0116435, CHE-0521063, CHE-0849677, and CHE-1229354.

\section{Notes and references}

1 G. Meller and T. Grasser, Organic Electronics, Springer, 2009. 2 T. A. Skotheim and J. R. Reynolds, Handb. Conduct. Polym., London, Boca Raton, Fla, 2007.

3 (a) D. Hu, J. Yu, K. Wong, B. Bagchl, P. J. Rossky and P. F. Barbara, Nature, 2000, 405, 1030; (b) T. Huser, M. Yan and L. J. Rothberg, Proc. Natl. Acad. Sci. U. S. A., 2000, 97, 11187.

4 (a) R. E. Martin and F. Diederich, Angew. Chem., Int. Ed., 1999, 38, 1350; (b) S. Ellinger, K. R. Graham, P. Shi, R. T. Farley, T. T. Steckler, R. N. Brookins, P. Taranekar, J. Mei, L. A. Padilha, T. R. Ensley, H. Hu, S. Webster, D. J. Hagan, E. W. Van Stryland, K. S. Schanze and J. R. Reynolds, Chem. Mater., 2011, 23, 3805; (c) S. S. Zade, N. Zamoshchik and M. Bendikov, Acc. Chem. Res., 2010, 44, 14; (d) R. E. Martin and F. Diederich, Angew. Chem., Int. Ed., 1999, 38, 1350.

5 (a) Y. Sun, G. C. Welch, W. L. Leong, C. J. Takacs, G. C. Bazan and A. J. Heeger, Nat. Mater., 2012, 11, 44; (b) O. Gidron, Y. Diskin-Posner and M. Bendikov, J. Am. Chem. Soc., 2010, 132, 2148; (c) F. Zhang, D. Wu, Y. Xu and X. Feng, J. Mater. Chem., 2011, 21, 17590; (d) O. Gidron, A. Dadvand, Y. Sheynin, M. Bendikov and D. F. Perepichka, Chem. Commun., 2011, 47, 1976.

6 (a) Q. Zhang, B. Kan, F. Liu, G. Long, X. Wan, X. Chen, Y. Zuo, W. Ni, H. Zhang, M. Li, Z. Hu, F. Huang, Y. Cao, Z. Liang, M. Zhang, T. P. Russell and Y. Chen, Nat. Photonics, 2015, 9, 35; (b) X. Liu, Y. Sun, B. B. Y. Hsu, A. Lorbach, L. Qi, A. J. Heeger and G. C. Bazan, J. Am. Chem. Soc., 2014, 136, 5697.

7 C. J. Tonzola, A. P. Kulkarni, A. P. Gifford, W. Kaminsky and S. A. Jenekhe, Adv. Funct. Mater., 2007, 17, 863.

8 M. Cai, T. Xiao, E. Hellerich, Y. Chen, R. Shinar and J. Shinar, Adv. Mater., 2011, 23, 3590.

9 (a) J. N. Wilson, M. Josowicz, Y. Wang and U. H. F. Bunz, Chem. Commun., 2003, 2962; (b) A. J. Zucchero, P. L. McGrier and U. H. F. Bunz, Acc. Chem. Res., 2009, 43, 397; (c) P. L. McGrier, K. M. Solntsev, S. Miao, L. M. Tolbert, O. R. Miranda, V. M. Rotello and U. H. F. Bunz, Chem. - Eur. J., 2008, 14, 4503; (d) J. A. Marsden, J. J. Miller, L. D. Shirtcliff and M. M. Haley, J. Am. Chem. Soc., 2005, 127, 2464; (e) E. L. Spitler, L. D. Shirtcliff and M. M. Haley, J. Org. Chem., 2007, 72, 86; 
$(f)$ E. L. Spitler, J. M. Monson and M. M. Haley, J. Org. Chem., 2008, 73, 2211; $(g)$ K. Ohta, S. Yamada, K. Kamada, A. D. Slepkov, F. A. Hegmann, R. R. Tykwinski, L. D. Shirtcliff, M. M. Haley, P. Salek, F. Gel'mukhanov and H. Aagren, J. Phys. Chem. A, 2011, 115, 105; (h) H. Kang, G. Evmenenko, P. Dutta, K. Clays, K. Song and T. J. Marks, J. Am. Chem. Soc., 2006, 128, 6194.

10 (a) J. E. Klare, G. S. Tulevski and C. Nuckolls, Langmuir, 2004, 20, 10068; (b) J. E. Klare, G. S. Tulevski, K. Sugo, A. de Picciotto, K. A. White and C. Nuckolls, J. Am. Chem. Soc., 2003, 125, 6030; (c) B. C. Tlach, A. L. Tomlinson, A. G. Ryno, D. D. Knoble, D. L. Drochner, K. J. Krager and M. Jeffries-El, J. Org. Chem., 2013, 78, 6570; (d) K. Osowska and O. S. Miljanic, Chem. Commun., 2010, 46, 4276; (e) J. Lim, T. A. Albright, B. R. Martin and O. Š. Miljanić, J. Org. Chem., 2011, 76, 10207; $(f)$ M. A. Saeed, H. T. M. Le and O. Š. Miljanić, Acc. Chem. Res., 2014, 47, 2074; $(g)$ M. Jo, J. Lim and O. Š. Miljanić, Org. Lett., 2013, 15, 3518; (h) J. Lim, D. Nam and O. S. Miljanic, Chem. Sci., 2012, 3, 559.

11 (a) B. C. Tlach, A. L. Tomlinson, K. D. Morgan, C. R. Collins, M. D. Zenner and M. Jeffries-El, Aust. J. Chem., 2014, 67, 711; (b) J. J. Intemann, E. S. Hellerich, B. C. Tlach, M. D. Ewan, C. A. Barnes, A. Bhuwalka, M. Cai, J. Shinar, R. Shinar and M. Jeffries-El, Macromolecules, 2012, 45, 6888.
12 (a) K. T. Kamtekar, A. P. Monkman and M. R. Bryce, Adv. Mater., 2010, 22, 572; (b) G. Schwartz, S. Reineke, T. C. Rosenow, K. Walzer and K. Leo, Adv. Funct. Mater., 2009, 19, 1319.

13 (a) J. Chen, C. Shi, Q. Fu, F. Zhao, Y. Hu, Y. Feng and D. Ma, J. Mater. Chem., 2012, 22, 5164; (b) M. Zhu and C. Yang, Chem. Soc. Rev., 2013, 42, 4963.

14 X. Yang, X. Xu and G. Zhou, J. Mater. Chem. C, 2015, 3, 913. 15 C. M. Cardona, W. Li, A. E. Kaifer, D. Stockdale and G. C. Bazan, Adv. Mater., 2011, 23, 2367.

16 D. Wheeler and A. e. L. Tomlinson, unpublished results.

17 (a) V. Barone and M. Cossi, J. Phys. Chem. A, 1998, 102, 1995; (b) M. Cossi, N. Rega, G. Scalmani and V. Barone, J. Comput. Chem., 2003, 24, 669.

18 Y. Yu, Z. Wu, Z. Li, B. Jiao, L. Li, L. Ma, D. Wang, G. Zhou and X. Hou, J. Mater. Chem. C, 2013, 1, 8117.

19 E. S. Hellerich, J. J. Intemann, M. Cai, R. Liu, M. D. Ewan, B. C. Tlach, M. Jeffries-EL, R. Shinar and J. Shinar, J. Mater. Chem. C, 2013, 1, 5191.

20 S.-K. Kim, B. Yang, Y. Ma, J.-H. Lee and J.-W. Park, J. Mater. Chem., 2008, 18, 3376.

21 B. C. Tlach, A. L. Tomlinson, A. Bhuwalka and M. Jeffries-El, J. Org. Chem., 2011, 76, 8670.

22 K. Yamamoto and H. Watanabe, Chem. Lett., 1982, 1225.

23 A. M. Brouwer, Pure Appl. Chem., 2011, 83, 2213-2228. 